\title{
Conversion of Beet Molasses and Cheese Whey into Fatty Acid Methyl Esters by the Yeast Cryptococcus curvatus
}

\author{
Naoya Takakuwa* and Katsuichi Saito \\ Memuro Research Station, National Agricultural Research Center for Hokkaido Region, National Agriculture and Food Research \\ Organization (Memuro, Kasai, Hokkaido 082-0081, JAPAN)
}

\begin{abstract}
Eighty-one yeast isolates from raw milk were surveyed for the production of fatty acid methyl esters (FAME). Only one species, identified as Cryptococcus curvatus, produced FAME at a detectable level. Cr. curvatus TYC-19 produced more FAME from beet molasses and cheese whey medium than other strains of the same species. In both media, the major FAME produced were linoleic and oleic acid methyl esters. Sequence analysis of the internal transcribed spacer region of ribosomal DNA indicated that TYC19 diverged from the same species.
\end{abstract}

Key words: fatty acid methyl ester, beet molasses, cheese whey; Cryptococcus curvatus

\section{INTRODUCTON}

Fatty acid methyl esters (FAME) are used worldwide as a biodiesel fuel. FAME are obtained using a transesterification process by adding methanol to triacylglycerol (TG) in the presence of an alkali, acid or lipase as catalyst ${ }^{1)}$. TG, the raw material of FAME, is found in oleaginous plants and is the predominant component of plant oils. The main production areas of typical oleaginous plants are the United States, EU countries and Southeast Asian countries, which grow soybean, rapeseed and palm, respectively. Therefore, the raw materials for FAME can be produced in large quantities from the oleaginous plants in each of these areas ${ }^{2)}$.

In Japan, however, it is difficult to cultivate oleaginous plants on a large scale and almost all FAME are produced from waste plant oils. Moreover, the supply of waste plant oils is inconsistent. Additionally, a complex manufacturing process is needed to obtain FAME of constant quality from waste plant oils, as they are inconsistent mixtures of TG from several types of oleaginous plants. In Japan, most plant oil is imported; therefore, the production of FAME from waste plant oils cannot be considered a self-sufficient process. In promoting the production of Japanese FAME, the technical challenge is to invent new manufacturing methods that use cheap raw materials originating in Japan and create a product of consistent quality.

Yeasts are a promising raw material for TG, although yields differ between species and strains ${ }^{3)}$. Cryptococcus curvatus (formerly Candida curvata and Apiotrichum curvatum) ${ }^{4)}$ and Rhodosporidum toruloides (formerly Rhodotorula gracilis) $)^{5)}$, known as oleaginous yeasts, usually synthesize TG using sugar as the substrate. Intracellular TG can be efficiently extracted using an organic solvent, such as methanol ${ }^{6)}$. On the other hand, yeasts such as Candida rugosa (formerly Ca. cylindracae $)^{7}$, Ca. parapsilosis ${ }^{8)}$ and Yarrowia lipolytica (formerly Ca. lipolytica) ) $^{9}$ produce large quantities of lipases that can catalyze the conversion of plant oils to FAME $^{10)}$.

The presence of yeasts that produce TG or lipase in raw milk and dairy products has been well documented ${ }^{11,12)}$. Unfortunately, the existence of yeast that produces large quantities of both TG and lipase has not been reported until now. In our opinion, the existence of such yeast would allow for the production of FAME by homogenizing the cells after adding methanol to the cultured yeast. Specifically, FAME would be produced by transesterification concurrent with the extraction of TG and lipase from cells.

\footnotetext{
* Correspondence to: Naoya Takakuwa, Memuro Research Station, National Agricultural Research Center for Hokkaido Region, National Agriculture and Food Research Organization, Memuro, Kasai, Hokkaido 082-0081, JAPAN

E-mail: takakuwa@naro.affrc.go.jp

Accepted December 21, 2009 (received for review November 9, 2009)

Journal of Oleo Science ISSN 1345-8957 print / ISSN 1347-3352 online

http://www.jstage.jst.go.jp/browse/jos/
} 


\section{N. Takakuwa and K. Saito}

Beet molasses and whey are by-products of the sugar and cheese industries, respectively. The principal sugars present in these by-products are sucrose in beet molasses and lactose in cheese whey ${ }^{13)}$. If these can be used as a growth medium, it would be possible to supply FAME of consistent quality at low cost. In the present study, we sought yeasts that could produce FAME by homogenizing the cells after the addition of methanol to cultured yeast, and assessed the production of FAME using beet molasses and cheese whey.

\section{EXPERIMENTAL}

\subsection{Yeast strains and culture}

Raw milk samples were obtained from dairy farms in three different parts (Asahikawa, Kamikawa, and Nakagawa) of Hokkaido, Japan. Aliquots of raw milk samples were spread on the following medium (wt/v): $1 \%$ yeast extract, $2 \%$ polypeptone, $2 \%$ glucose, $0.1 \%$ chloramphenicol, and $2 \%$ agar. All components of the media were obtained from Wako Pure Chemical Industries (Osaka, Japan). After incubation for two days, 81 yeast-like colonies were isolated. The isolates were identified by phenotypic tests such as filamentous growth, ballistoconidia, ascospores and the ability to use organic compounds as the sole source of carbon ${ }^{14}$. When necessary, test results were checked using the API ID 32C (bioMerieux, Tokyo, Japan). Oleaginous yeasts (Cr. curvatus ATCC 20509 (Ca. curvata D) $)^{4}$ and $R$. toruloides NBRC 0559 (NRRL Y-1091) $\left.{ }^{5}\right)$, lipase-producing yeasts (Ca. cylindracea CBS 6330 (NRRL Y-17506) ${ }^{7)}$, Ca. parapsilosis NBRC 1396 (CBS 604) and Y. lipolytica CBS 7504 $\left(\right.$ ATCC 20460) ${ }^{9}$ ) and thirteen strains of $C r$. curvatus (Table 1) were obtained from ATCC (American Type Culture Collection, Manassas, VA, USA), CBS (Centraalbureau voor Schimmelcultures, Baarn, Netherlands) and NBRC (NITE Biological Research Center, Chiba, Japan).

The growth medium was comprised of (wt/v): $1 \%$ yeast extract, $2 \%$ polypeptone, and $2 \%$ carbon source. The carbon sources used were: glucose, sucrose (as beet molasses), and lactose (as cheese whey). All cultures were performed in 200-mL baffled shake flasks that contained $40 \mathrm{~mL}$ of each growth medium. The flasks were incubated at $30^{\circ} \mathrm{C}$ for $24 \mathrm{~h}$ and shaken at $200 \mathrm{rpm}$.

\subsection{FAME analysis}

Cell mass was estimated from freeze dried cells. The culture medium $(10 \mathrm{~mL})$ was centrifuged, washed twice with distilled water and freeze-dried overnight.

Culture samples $(30 \mathrm{~mL})$ were also centrifuged and washed twice with distilled water. The pellets were treated in $20 \mathrm{~mL}$ of methanol for $3 \mathrm{~min}$ by an ultrasonic homogenizer (Kenis Co., Osaka, Japan) and incubated at $30^{\circ} \mathrm{C}$ for $18 \mathrm{~h}$ with shaking $(200 \mathrm{rpm})$. After further mixing with $20 \mathrm{~mL}$ of hexane and centrifugation, the hexane phase was dried on a rotary evaporator and the residue was re-dissolved in 100 $\mu \mathrm{L}$ of hexane.

The concentrations of FAME and TG were measured by thin layer chromatography (TLC) from $5 \mu \mathrm{L}$ of the extract spotted on a TLC aluminum sheet Silica gel 60 (Merck) and developed using hexane:diethyl ether:acetic acid (80:20:1, $\mathrm{v} / \mathrm{v}) .50 \%(\mathrm{v} / \mathrm{v}) \mathrm{H}_{2} \mathrm{SO}_{4}$ was used to visualize the spots of FAME and TG, which were quantified by comparing densities to those of an authentic standards with a Lane and Spot Analyzer (Atto Co., Tokyo, Japan).

FAME recovered from TLC was analyzed by a gas chromatograph (GC-2010, Shimadzu, Kyoto, Japan) connected to an FID detector using a capillary column (CP-Sil 88). Injector and detector temperatures were 230 and $240^{\circ} \mathrm{C}$, respectively. The column temperature program was as follows: a starting temperature of $80^{\circ} \mathrm{C}$, increasing to $160^{\circ} \mathrm{C}$ at $10^{\circ} \mathrm{C} / \mathrm{min}$, with subsequent heating to $220^{\circ} \mathrm{C}$ at $2^{\circ} \mathrm{C} / \mathrm{min}$, which was held for 2 min. A variety of fatty acids were identified from a mixed FAME standard (Matreya Inc., Pleasant Gap, PA, USA).

Analyses of all the samples were carried out in triplicate and average values were reported.

\subsection{Molecular phylogenetic analysis}

The internal transcribed spacer (ITS) of the rRNA gene was amplified by PCR as described previously ${ }^{15)}$. The DNA fragments were sequenced with an automated DNA sequencer (Model 310, Applied Biosystems, Foster City, CA, USA). A phylogenetic tree was constructed using the CLUSTAL W and TreeView programs (http://taxonomy.zoology.gla.ac.uk/rod/treeview.html). The nucleotide sequences for ITS have been assigned EMBL/GenBank/DDBJ accession numbers AB520683 to AB520686.

\section{RESULTS AND DISCUSSION}

The 81 yeast strains were isolated from raw milk samples. Using phenotypic tests, the strains were classified into ten groups. The species and number of strains were: Ca. catemulata, 2; Ca. inconspicua, 1; Ca. parapsilosis, 8; Ca. zeylanoides, 3; Cr. curvatus, 29; Debaryomces hansenii, 2; Issatchenkia orientalis, 2; Pichia anomala, 3; $P$. fermentans, 11; and Y. lipolytica, 20. All strains from each species were cultured in medium with glucose as the carbon source. The production of FAME by isolated yeast strains was compared to those of $\mathrm{Cr}$. curvatus ATCC 20509, R. toruloides NBRC 0559, Ca. cylindracea CBS 6330, Ca. parapsilosis NBRC 1396, and Y. lipolytica CBS 7504. FAME were detected in abundance only from $\mathrm{Cr}$. curvatus and were not detected, or detected in only minute amounts, in other yeasts. The production of FAME did not differ between the same species. TLC analysis of methanol 
extracts of a representative strain of each species is shown in Fig. 1. Cr. curvatus is known to copiously synthesize intracellular $\mathrm{TG}^{4}$. Within the genus Cryptococcus, lipase activity differs among species and strains, but a wide range of species that produce lipase have been found ${ }^{16,17}$. Therefore, it is thought that the isolates were able to produce FAME from TG due to high levels of lipase expression and/or expressing highly active lipase in cells.

TYC-19, which is a representative strain was cultured in media using beet molasses and cheese whey as carbon sources and compared to the 13 other strains of $\mathrm{Cr}$. curvatus (Table 1). All of the strains grew well on both media, but strain TYC-19 was the only strain with markedly detectable levels of FAME. Strain TYC-19 produced 68.3 $\mathrm{mg} / \mathrm{L}$ FAME in the beet molasses medium and $78.8 \mathrm{mg} / \mathrm{L}$ in the cheese whey medium. Moreover, the transesterification rate from TG to FAME was extremely high (more than 95\%) in both media. From these results, strain TYC-19 appeared to be the most suitable strain for the efficient production of FAME from beet molasses and cheese whey.

For further taxonomical analysis of strain TYC-19, the ITS sequences, which appears to be a powerful technique to differentiate between phylogenetically intimately related species ${ }^{18)}$, were determined. These results revealed that the strains were divided into three groups and strain TYC19 branched off independently from all strains (Fig. 2). This suggests that strain TYC-19 differs genetically from other Cr. curvatus strains.

Table 2 compares the composition of FAME produced by strain TYC-19 when cultured in a beet molasses or cheese whey medium. In both media, the principal FAME were linoleic acid methyl ester and oleic acid methyl ester. Additionally, in the composition of other FAME, there was no great difference between either culture medium. Therefore, FAME of identical composition may be stably produced using either beet molasses or cheese whey as carbon sources.

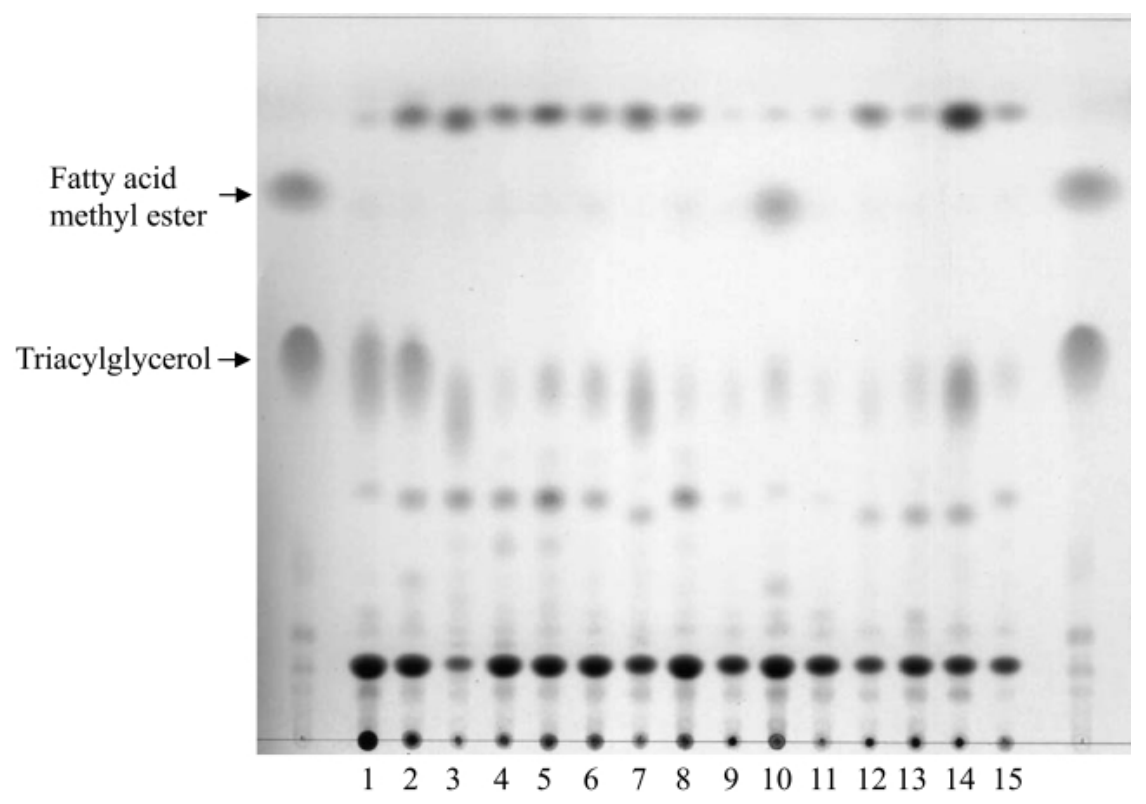

Fig. 1 TLC Analysis of Methanol Extracts from Various Yeast Strains.

The plate was developed with hexane:diethyl ether:acetic acid (80:20:1, $\mathrm{v} / \mathrm{v})$ and heated after spraying with $50 \%(\mathrm{v} / \mathrm{v}) \mathrm{H}_{2} \mathrm{SO}_{4}$. The lanes at both ends represent the authentic standards of FAME and TG at $50 \mu \mathrm{g}$, respectively. Samples: 1, Cryptococcus curvatus ATCC 20509; 2, Rhodosporidium toruloides NBRC 0559; 3, Candida cylindracea CBS 6330; 4, Candida parapsilosis NBRC 1396; 5, Yarrowia lipolytica CBS 7504; 6, Candida catenulate TYC-1; 7, Candida inconspicua TYC-3; 8, Candida parapsilosis TYC-4; 9, Candida zeylanoides TYC-12; 10, Cryptococcus curvatus TYC-19; 11, Debaryomyces hansenii TYC-44; 12, Issatchenkia orientalis TYC-46; 13, Pichia anomala TYC-48; 14, Pichia fermentans TYC-51; 15, Yarrowia lipolytica TYC-81. Samples 1 to 5 are reference strains, and samples 6 to 15 are yeast strains isolated from raw milk. 


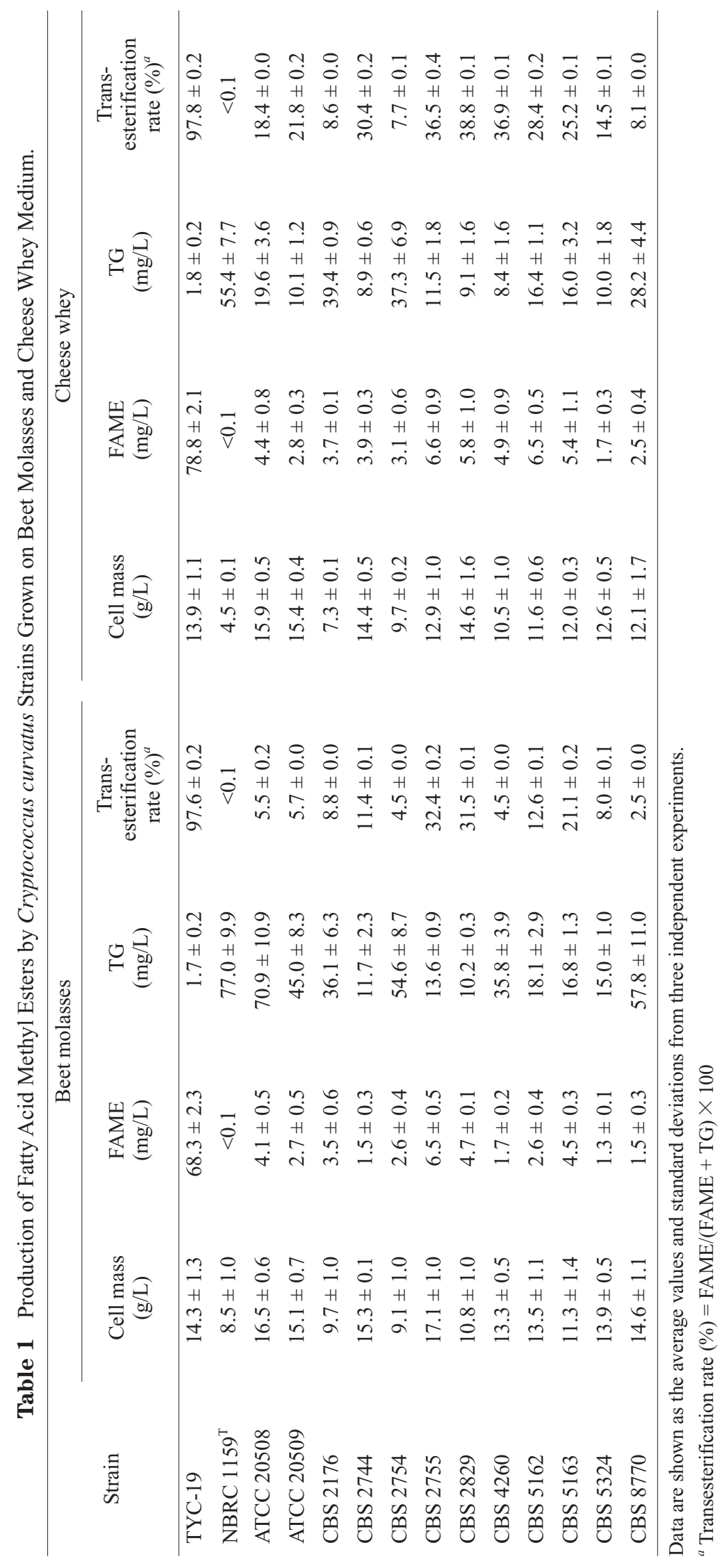




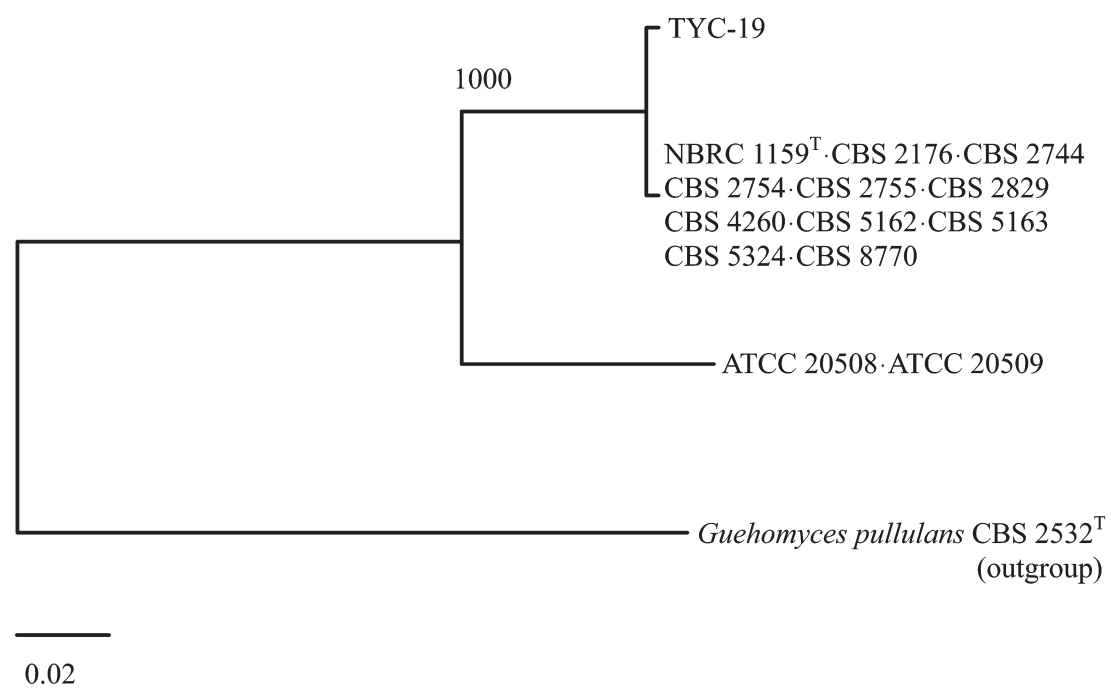

Fig. 2 Phylogenetic Tree Constructed by the Neighbor-Joining Method from ITS Sequences of Cryptococcus curvatus Strains.

ITS sequences of CBS 8770 (AB520683), ATCC 20508 (AB520684), ATCC 20509 (AB520685) and TYC-19 (AB520686) were determined in this study. The retrieved sequences were NBRC $1159^{\mathrm{T}}\left(\mathrm{JCM} 1532^{\mathrm{T}}\right)(\mathrm{AB} 035574), \mathrm{CBS}$ 2176 (AB035688), CBS 2744 (AB035689), CBS 2754 (AB035690), CBS 2755 (AB035691), CBS 2829 (AB035692), CBS 4260 (AB035693), CBS 5162 (AB035694), CBS 5163 (AB035695), CBS 5324 (AB035696) and Guehomyces pullulans (formerly Trichosporon pullulans) CBS $2532^{\mathrm{T}}$ (AF444417) as the outgroup. The bar indicates one estimated substitution per 100 nucleotide positions. Bootstrap values were calculated from 1,000 trees.

Table 2 Composition (wt\%) of Fatty Acid Methyl Ester from Cr. curvatus TYC-19 Grown on Beet Molasses and Cheese Whey Medium.

\begin{tabular}{lcc}
\hline \multicolumn{1}{c}{ Fatty acid } & Beet molasses & Cheese whey \\
\hline Palmitic (C16:0) & $3.5 \pm 0.7$ & $3.4 \pm 0.3$ \\
Palmitoleic (C16:1) & $<0.1$ & $0.4 \pm 0.1$ \\
Stearic (C18:0) & $<0.1$ & $0.7 \pm 0.1$ \\
Oleic (C18:1) & $41.2 \pm 5.7$ & $43.2 \pm 3.0$ \\
Linoleic (C18:2) & $48.1 \pm 3.6$ & $46.4 \pm 3.6$ \\
Linolenic (C18:3) & $7.2 \pm 1.4$ & $5.9 \pm 0.8$ \\
\hline
\end{tabular}

Data are shown as the average values and standard deviations from three independent experiments.

In Japan, sugar and cheese manufacturing plants produce large quantities of molasses and whey, estimated to be 15,000 tons ( $50 \%$ sucrose content) and 157,000 tons (5\% lactose content) annually, respectively. A similar amount of sugar is present in both by-products. Therefore, the two production methods will complement each other and contribute a stable supply of FAME. The yield of TG in $\mathrm{Cr}$. curvatus tends to increase with a high carbon/nitrogen ratio $\left(\mathrm{C} / \mathrm{N}\right.$ ratio) in the medium ${ }^{19)}$. If these culture conditions are applied to strain TYC-19, it will likely be possible to produce large amounts of FAME.

\section{CONCLUSION}

In the present study, we found it was possible to efficiently produce FAME from $\mathrm{Cr}$. curvatus TYC-19, using beet molasses and cheese whey as culture medium and by homogenizing the cells after the addition of methanol to the cultured cells. It will be necessary to study in detail culture conditions, such as the $\mathrm{C} / \mathrm{N}$ ratio in the culture medium, to increase the production of FAME.

\section{ACKNOWLEDGEMENTS}

This work was supported by the Program for Promotion of Basic Research Activities for Innovative Biosciences (PROBRAIN). 


\section{N. Takakuwa and K. Saito}

\section{References}

1. Bajpai, D.; Tyagi, V.K. Biodiesel: source, production, composition, properties and its benefits. J. Oleo Sci. 55, 487-502 (2006).

2. Shay, E.G. Diesel fuel from vegetable oils: Status and opportunities. Biomass Bioenerg. 4, 227-242 (1993).

3. Ratledge, C; Wynn, J.P. The biochemistry and molecular biology of lipid accumulation in oleaginous microorganisms. Adv. Appl. Microbiol. 51, 1-51 (2002).

4. Evans, C.T.; Ratledge, C. A comparison of the oleaginous yeast, Candida curvata, grown on different carbon sources in continuous and batch culture. Lipids 18, 623-629 (1983).

5. Yoon, S.H.; Rhim, J.W.; Choi, S.Y.; Ryu, D.D.W.; Rhee, J.S. Effect of carbon and nitrogen sources on lipid production of Rhodotorula gracilis. J. Ferment. Technol. 60, 243-246 (1982).

6. Moon, N.J.; Hammond, E.G. Oil production by fermentation of lactose and the effect of temperature on the fatty acid composition. J. Am. Oil Chem. Soc. 55, 683688 (1978).

7. Kim, B.S.; Hou, C.T. Production of lipase by high cell density fed-batch culture of Candida cylindracea. Bioprocess Biosyst. Eng. 29, 59-64 (2006).

8. Neugnot, V.; Moulin, G.; Dubreucq, E.; Bigey, F. The lipase/acyltransferase from Candida parapsilosis: Molecular cloning and characterization of purified recombinant enzymes. Eur. J. Biochem. 269, 17341745 (2002).

9. Lopes, M.; Gomes, N.; Gonçalves, C.; Coelho, M.A.; Mota, M.; Belo, I. Yarrowia lipolytica lipase production enhanced by increased air pressure. Lett. Appl. Microbiol. 46, 255-260 (2008).

10. Kaieda, M.; Samukawa, T.; Kondo, A.; Fukuda, H. Effect of methanol and water contents on production of biodiesel fuel from plant oil catalyzed by various lipases in a solvent-free system. J. Biosci. Bioeng. 91, 12-15 (2001).

11. Corbo, M.R.; Lanciotti, R.; Albenzio, M.; Sinigaglia, M.
Occurrence and characterization of yeasts isolated from milks and dairy products of Apulia region. Int. J. Food Microbiol. 69, 147-152 (2001).

12. Prillinger, H.; Molnár, O.; Eliskases-Lechner, F.; Lopandic, K. Phenotypic and genotypic identification of yeasts from cheese. Antonie Van Leeuwenhoek 75, 267-283 (1999).

13. Josh, S.; Bharucha, C.; Jha, S.; Yadav, S.; Nerurkar, A.; Desai, A.J. Biosurfactant production using molasses and whey under thermophilic conditions. Bioresour. Technol. 99, 195-199 (2008).

14. Kurtzman, C.P.; Fell, J.W. The yeast: A taxonomic study. $4^{\text {th }}$ ed. Elsevier. Amesterdam. pp. 80-98 (1998).

15. Sugai, M.; Takakuwa, N.; Ohnishi, M.; Arai, I.; Urashima, T.; Oda, Y. Selection of lactic yeast producing glucosylceramide from cheese whey. Bioresour. Technol. 98, 3643-3646 (2007).

16. Brizzio, S.; Turchetti, B.; de García, V.; Libkind, D.; Buzzini, P.; van Broock, M. Extracellular enzymatic activities of basidiomycetous yeasts isolated from glacial and subglacial waters of northwest Patagonia (Argentina). Can. J. Microbiol. 53, 519-525 (2007).

17. Kamini, N.R.; Fujii, T.; Kurosu, T.; Iefuji, H. Production, purification and characterization of an extracellular lipase from the yeast Cryptococcus sp. S-2. Process Biochem. 36, 317-324 (2000).

18. Sugita, T.; Takashima, M.; Ikeda, R.; Nakase, T; Shino$\mathrm{da}, \mathrm{T}$. Phylogenetic and taxonomic heterogeneity of Cryptococcus humicolus by analysis of the sequences of the internal transcribed spacer regions and $18 \mathrm{~S}$ rDNA, and the phylogenetic relationships of C. humicolus, C. curvatus, and the genus Trichosporon. Microbiol. Immunol. 44, 455-461 (2000).

19. Hassan, M.; Blanc, P.J.; Granger, L.M.; Pareilleux, A.; Goma, G. Influence of nitrogen and iron limitations on lipid production by Cryptococcus curvatus grown in batch and fedbatch culture. Process Biochem. 31, 355-361 (1996). 